\title{
Nutritional screening, assessment and provision of advice for people living with and beyond cancer - a UK survey of clinicians
}

\author{
B.M. Corfe, J.L. Murphy, F.P. Davey, L.J. Miller, M.A. Lloyd, S. Burden, F. Munir, \\ T. Wiseman, M. Barrett and S.A. Wootton \\ Cancer and Nutrition NIHR infrastructure collaboration, NIHR Southampton Biomedical Research Centre, University \\ Hospital Southampton NHS Foundation Trust Southampton SO16 6 YD.
}

People diagnosed with cancer often experience nutrition-related problems and they do not receive consistent evidence-based advice ${ }^{(1)}$. Studies indicate that just $30-60 \%$ of cancer patients who were at risk of malnutrition received nutritional support ${ }^{(2)}$. The National Institute for Health Research Cancer and Nutrition infrastructure collaboration (http://cancerandnutrition.nihr.ac.uk) sought to determine the breadth and diversity of practice across professional groups in identifying and assessing the need for nutritional support. We report the findings of a survey aimed at understanding clinicians' awareness and current practice for screening and assessing nutritional status, the provision of nutrition advice and use of relevant nutrition support guidelines, and training needs.

A national cross-sectional online survey comprising 21 questions was sent to health care professionals via professional groups, networks and personal contacts. Of the 610 responses received, there were 191 nurses $(31 \%), 152$ dietitians $(25 \%), 187$ doctors $(31 \%)$ and 54 Speech and Language Therapists (9\%) and 26 from 'other'.

The majority of respondents $(77 \%)$ indicated that they used a screening tool to identify people at risk of malnutrition in the in-patient settings but only $29 \%$ reported screening for malnutrition in the out-patient setting. The Malnutrition Universal Screening Tool was the most widely reported tool for screening, used by $68 \%$ of dietitians, $61 \%$ of nurses and $39 \%$ of doctors for inpatient settings. Overall, $33 \%$ of respondents systematically collect nutritional data of their cancer patients, with dietitians most likely to do so $(64 \%)$. Of those that do collect data, the principle routine measurements were weight, height and BMI $(81 \%)$, weight loss $(63 \%)$, appetite $(82 \%)$ and risk of poor intake $(62 \%)$. The most common concerns raised with health professionals were loss of appetite, nausea/ vomiting, weight loss, change in taste/smell, constipation and fatigue. Dietitians reported that they all discuss nutrition with their patients, had been trained $(80 \%)$ and were completely confident $(70 \%)$ in providing advice on nutritional care in cancer and were aware of recent guidelines (79\%). In contrast, although doctors (88 \%) and nurses (96\%) discussed nutritional concerns with their patients, few were completely confident in giving that advice (doctors $2 \%$, nurses $4 \%$ ) and most had not received any formal training in nutritional care and were generally unaware of guidelines for the nutritional care of cancer patients (doctors $81 \%$ and nurses $70 \%$ ). There was a perceived need for further training in relation to nutrition and cancer from all groups (dietitians $48 \%$; doctors $66 \%$, nurses $81 \%$ ) particularly on dietary advice for specific cancers and cancer stage (70 \%), assessment of nutritional status $(63 \%)$, alternative dietary approaches $(57 \%)$ and dietary supplements $(47 \%)$.

These findings show that whilst health professionals discuss nutritional matters with cancer patients and provide information or advice to people living with and beyond cancer, many lack relevant training and awareness of current guidelines. There is scope for the provision of further training for clinicians on nutrition and cancer to ensure both consistency in practice and improved patient care.

(*Presented in National Institute for Health Research session 12.00-13.00 on Wednesday 6 December.)

1. Beeken RJ, Williams K, Wardle J et al. (2016) Eur J Cancer Care. 25, 774-83.

2. Arends J, Baracos V, Bertz H et al. (2017) Clin Nutr. 36, 1187-96. 